\title{
Negative-pressure-induced helimagnetism in ferromagnetic cubic perovskites $\mathrm{Sr}_{1-x} \mathrm{Ba}_{x} \mathrm{CoO}_{3}$
}

\author{
H. Sakai, ${ }^{1,2,3,{ }^{*}}$ S. Yokoyama, ${ }^{1}$ A. Kuwabara, ${ }^{1}$ J. S. White, ${ }^{4}$ E. Canévet, ${ }^{4,5}$ H. M. Rønnow,,${ }^{6,7}$ T. Koretsune,${ }^{8}$ R. Arita, ${ }^{1,7}$ \\ A. Miyake, ${ }^{9}$ M. Tokunaga, ${ }^{9}$ Y. Tokura,${ }^{1,7}$ and S. Ishiwata ${ }^{1,3,},{ }^{,}$ \\ ${ }^{1}$ Department of Applied Physics, University of Tokyo, Tokyo 113-8656, Japan \\ ${ }^{2}$ Department of Physics, Osaka University, Toyonaka, Osaka 560-0043, Japan \\ ${ }^{3}$ PRESTO, Japan Science and Technology Agency, Kawaguchi, Saitama 332-0012, Japan \\ ${ }^{4}$ Laboratory for Neutron Scattering and Imaging, Paul Scherrer Institut, Villigen CH-5232, Switzerland \\ ${ }^{5}$ Department of Physics, Technical University of Denmark, 2800 Kongens, Lyngby, Denmark \\ ${ }^{6}$ Laboratory for Quantum Magnetism, École Polytechnique Fédérale de Lausanne (EPFL), Lausanne CH-1015, Switzerland \\ ${ }^{7}$ RIKEN Center for Emergent Matter Science (CEMS), Wako 351-0198, Japan \\ ${ }^{8}$ Department of Physics, Tohoku University, Sendai 980-8578, Japan \\ ${ }^{9}$ The Institute for Solid State Physics, University of Tokyo, Kashiwa 277-8581, Japan
}

(Received 29 June 2018; published 26 October 2018)

\begin{abstract}
Helimagnetic materials are identified as promising for novel spintronic applications. Since helical spin order is manifested as a compromise of competing magnetic exchange interactions, its emergence is limited by unique constraints imposed by the crystalline lattice and the interaction geometries as exemplified by the multiferroic perovskite manganites with large orthorhombic distortion. Here we show that a simple cubic perovskite $\mathrm{SrCoO}_{3}$ with room-temperature ferromagnetism has the potential to host helimagnetic order upon isotropic lattice expansion. Increasing the $\mathrm{Ba}$ content $x$ in $\mathrm{Sr}_{1-x} \mathrm{Ba}_{x} \mathrm{CoO}_{3}$ continuously expands the cubic lattice, eventually suppressing the ferromagnetic order near $x=0.4$ where helimagnetic correlations are observed as incommensurate diffuse magnetic scattering by neutron-diffraction measurements. The emergence of helimagnetism is semiquantitatively reproduced by first-principles calculations, leading to the conjecture that a simple cubic lattice with strong $d$ - $p$ hybridization can exhibit a variety of novel magnetic phases originating from competing exchange interactions.
\end{abstract}

DOI: $10.1103 /$ PhysRevMaterials.2.104412

\section{INTRODUCTION}

The exploration of helimagnetic (HM) materials and the relevant novel quantum phases, such as multiferroic [1,2] and skyrmion lattice [3] phases, has been one of the hottest research topics in the field of condensed-matter physics as well as spintronics. For helimagnetic-based spintronics, perovskite-type transition-metal oxides can be promising candidates when finely controlling the interplay among spin, orbital, and lattice degrees of freedom. This is exemplified by the spin-spiral-driven ferroelectricity in perovskite-type manganites showing giant magnetoelectric effects $[4,5]$. The spinspiral orders in these manganites arise from the frustration of the exchange interactions between the $\mathrm{Mn}^{3+}$ spins, which is enhanced by the orthorhombic $\left(\mathrm{GdFeO}_{3}\right.$-type $)$ distortion with orbital order [6]. On the other hand, perovskite-type ferrites $A \mathrm{FeO}_{3}(A=\mathrm{Sr}, \mathrm{Ba})$ with unusually high-valence ions of $\mathrm{Fe}^{4+}$ show helical spin order even in a simple cubic lattice [7-11]. Recently, itinerant helimagnets with high lattice sym-

\footnotetext{
*Corresponding authors: sakai@phys.sci.osaka-u.ac.jp; ishiwata@ap.t.u-tokyo.ac.jp

Published by the American Physical Society under the terms of the Creative Commons Attribution 4.0 International license. Further distribution of this work must maintain attribution to the author(s) and the published article's title, journal citation, and DOI.
}

metry have been predicted to host a rich variety of topologically nontrivial magnetic structures typified by the skyrmion lattice [12-14]. Thus, cubic perovskite $A \mathrm{FeO}_{3}$ generates strong interest as a promising class of materials for novel topological magnets $[9,15]$.

The origin of the helimagnetic order in $\mathrm{SrFeO}_{3}$ is ascribable to a strong hybridization between the $\mathrm{Fe} 3 d$ and the $\mathrm{O} 2 p$ orbitals with the negative charge-transfer energy $\Delta$ yielding itinerant ligand holes [13,16,17]. Reflecting the correlation between the nature of the magnetic order and the $p$ - $d$ hybridization, the magnetic structure of $A \mathrm{FeO}_{3}$ is strongly dependent on the size of the $A$-site ion since this affects the bandwidth of the system. Although $\mathrm{SrFeO}_{3}$ and $\mathrm{BaFeO}_{3}$ share the same simple cubic structure, they show a different type of helical spin order [10,11]; the propagation vector of $\mathrm{BaFeO}_{3}$ is aligned with the $\langle 001\rangle$ direction, whereas that of $\mathrm{SrFeO}_{3}$ is aligned along the $\langle 1111\rangle$ direction.

Perovskite-type $\mathrm{ACoO}_{3}(A=\mathrm{Ca}, \mathrm{Sr})$ with the highvalence state of $\mathrm{Co}^{4+}$ is another interesting example of a negative- $\Delta$ system having itinerant ligand holes [18-23]. $\mathrm{SrCoO}_{3}$ has a cubic structure and shows metallic and ferromagnetic (FM) behaviors at ambient pressure with the ferromagnetic transition temperature of $T_{\mathrm{C}}=305 \mathrm{~K}$. Very recently, $\mathrm{CaCoO}_{3}$ was reported to be an antiferromagnetic metal with a distorted perovskite structure [23]. Thus, the sizes of the $A$-site ions emerge as dominant factors for the magnetic order in $A_{C o O}$ as well as for $A \mathrm{FeO}_{3}$. Furthermore, unlike the ferrites, cobaltates display a variability of the 


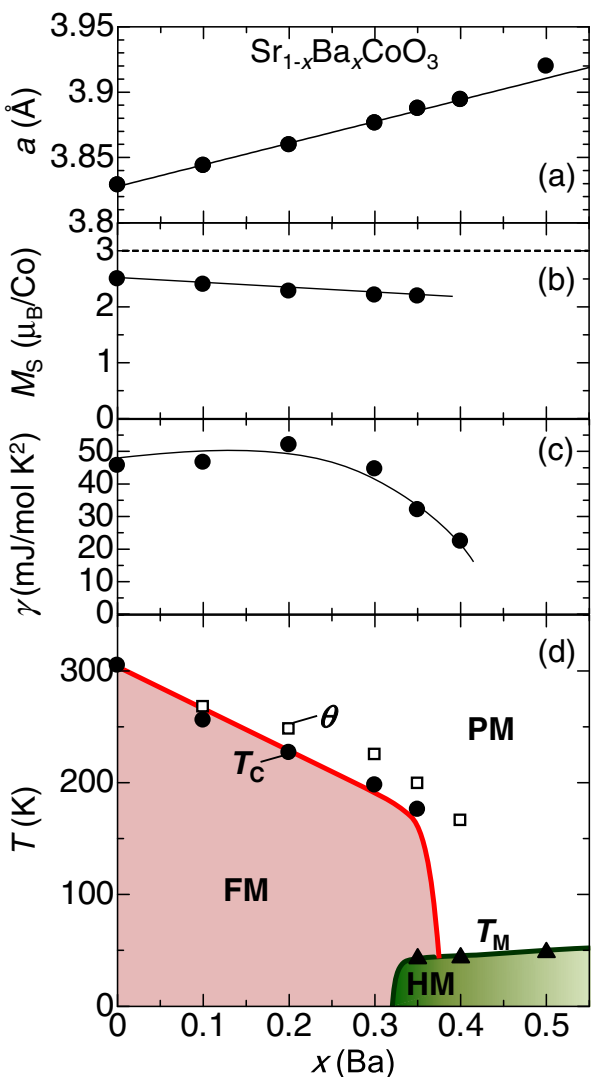

FIG. 1. $x$ dependence of the (a) cubic lattice constant $a$ at room temperature, (b) saturation magnetization $M_{\mathrm{S}}$ at the lowest temperature $(2-4 \mathrm{~K})$, and (c) electronic specific-heat coefficient $\gamma$ for $\mathrm{Sr}_{1-x} \mathrm{Ba}_{x} \mathrm{CoO}_{3}(0 \leqslant x \leqslant 0.4)$ single crystals. (d) The magnetic phase diagram as functions of temperature $T$ and $x$ where the FM and HM transition temperatures are denoted by closed circles $\left(T_{\mathrm{C}}\right)$ and closed triangles $\left(T_{\mathrm{M}}\right)$, respectively. The Weiss temperatures $\theta$ are also plotted as open squares. The data for $x=0$ are from Refs. [20,21].

spin states (high/intermediate/low) [24-26], which emerges as another factor influencing the magnetic order. In this paper, we explore novel magnetic order in the perovskite-type cobaltates with negative $\Delta$ and study the effect of lattice expansion on the ferromagnetic order in $\mathrm{SrCoO}_{3}$ by synthesizing singlecrystalline samples of $\mathrm{Sr}_{1-x} \mathrm{Ba}_{x} \mathrm{CoO}_{3}$ using a high-pressure technique ([9,21,27,28] and the Supplemental Material [29]). By increasing the Ba content, the ferromagnetic transition is suppressed monotonically and then disappears around $x \sim$ 0.4 yet all the while keeping the cubic lattice structure and intermediate spin state. Neutron-diffraction studies reveal that the compound with $x=0.4$ hosts short-range helimagnetic correlations described by an incommensurate propagation vector along the $\left\langle\begin{array}{llll}1 & 1 & 1\end{array}\right\rangle$ direction at the lowest temperature. We will discuss the origin of the emergent helimagnetic instability in $\mathrm{Sr}_{1-x} \mathrm{Ba}_{x} \mathrm{CoO}_{3}$ on the basis of first-principles calculations.

\section{EXPERIMENTAL AND COMPUTATIONAL DETAILS}

Single crystals of perovskite $\mathrm{Sr}_{1-x} \mathrm{Ba}_{x} \mathrm{CoO}_{3}(0.1 \leqslant x \leqslant$ $0.5)$ with cubic structure were synthesized through a two-step procedure $[9,21,27,28]$. We first synthesized single crystals of oxygen-deficient $\mathrm{Sr}_{1-x} \mathrm{Ba}_{x} \mathrm{CoO}_{3-\delta}(\delta \sim 0.5)$ by a floatingzone method as follows. Mixed powders of $\mathrm{SrCO}_{3}, \mathrm{BaCO}_{3}$, and $\mathrm{Co}_{3} \mathrm{O}_{4}$ in stoichiometric proportions were calcined at $900{ }^{\circ} \mathrm{C}$ for 12 hours in air. The resulting powders were pulverized and then pressed into a rod with $\sim 5 \mathrm{~mm}$ in diameter and $\sim 80 \mathrm{~mm}$ in length. The rod was fired at $1200^{\circ} \mathrm{C}$ for 24 hours in air, followed by water quenching down to room temperature. The crystal growth was performed at a rate of $1.5-3 \mathrm{~mm} / \mathrm{h}$ in an argon atmosphere while rotating the feed and seed rods in opposite directions. Second, pieces of the single crystals (typically $10-30 \mathrm{~mm}^{3}$ ) were sealed in a gold capsule with an oxidizing agent $\left(\mathrm{NaClO}_{3}\right)$ and were treated at $\sim 7.5 \mathrm{GPa}$ and $\sim 480^{\circ} \mathrm{C}$ for $1 \mathrm{~h}$ in a cubic anvil-type high-pressure apparatus. The obtained single crystals were fully-oxidized $\mathrm{Sr}_{1-x} \mathrm{Ba}_{x} \mathrm{CoO}_{3}(0.1 \leqslant x \leqslant 0.5)$ with a cubic structure. For details, see Fig. S1 of the Supplemental Material [29].

Resistivity was measured by a conventional four-terminal method with electrodes formed by room-temperature curing gold paste. Specific heat was measured with a Physical Properties Measurement System (Quantum Design). Low-field magnetization up to $7 \mathrm{~T}$ was measured with a superconducting quantum interference device (Quantum Design). High-field magnetization up to $\sim 40 \mathrm{~T}$ was measured by the induction method with coaxial pickup coils using the nondestructive pulsed magnet at the International Mega-Gauss Science Laboratory at the Institute for Solid State Physics. Neutron diffraction for a single crystal with $x=0.4$ was performed with the diffractometer DMC $(\lambda=3.8 \AA)$ at the Swiss Spallation Neutron Source (SINQ), Paul Scherrer Institut, Switzerland.

Electronic structure calculations were performed within the context of density functional theory using the PerdewBurke-Ernzerhof exchange-correlation functional [30] as implemented in the VASP code [31,32]. The projector augmented wave pseudopotentials with a cutoff energy of $500 \mathrm{eV}$ were used. A generalized Bloch theorem was adopted for the calculations of the spiral spin structures [33].

\section{RESULTS AND DISCUSSIONS}

The room-temperature structure was determined from $\mathrm{x}$ ray diffraction on powder obtained by grinding pieces of single crystals. For all $x$ 's, the diffraction profiles showed the cubic symmetry $(P m \overline{3} m)$ with the $x$-dependent lattice constant $a$ as depicted in Fig. 1(a). The value of $a$ increases linearly with $x$ from 0 to 0.4 in accordance with Vegard's law, whereas that for $x=0.5$ slightly deviates upward from the straight-line fit for the data in the range of $x \leqslant 0.4$. This slight increase in $a$ for $x=0.5$ may arise due to inevitable oxygen release after the high-pressure treatment, which is furthermore consistent with thermogravimetry measurements revealing a slight increase in the oxygen deficiency (see Fig. S1 in the Supplemental Material [29]). We note that $\mathrm{Sr}_{1-x} \mathrm{Ba}_{x} \mathrm{MnO}_{3}$ hosting a similar tolerance factor exhibits a structural transition from cubic to tetragonal symmetry for $x \geqslant 0.45$ [34], whereas $\mathrm{Sr}_{1-x} \mathrm{Ba}_{x} \mathrm{CoO}_{3}$ remains cubic up to $x=0.5$.

Figure 2(a) shows the temperature dependence of the magnetization $M$ at $0.01 \mathrm{~T}$ for $\mathrm{Sr}_{1-x} \mathrm{Ba}_{x} \mathrm{CoO}_{3}$, measured upon heating after a field-cooling process. With increasing $x$ from 

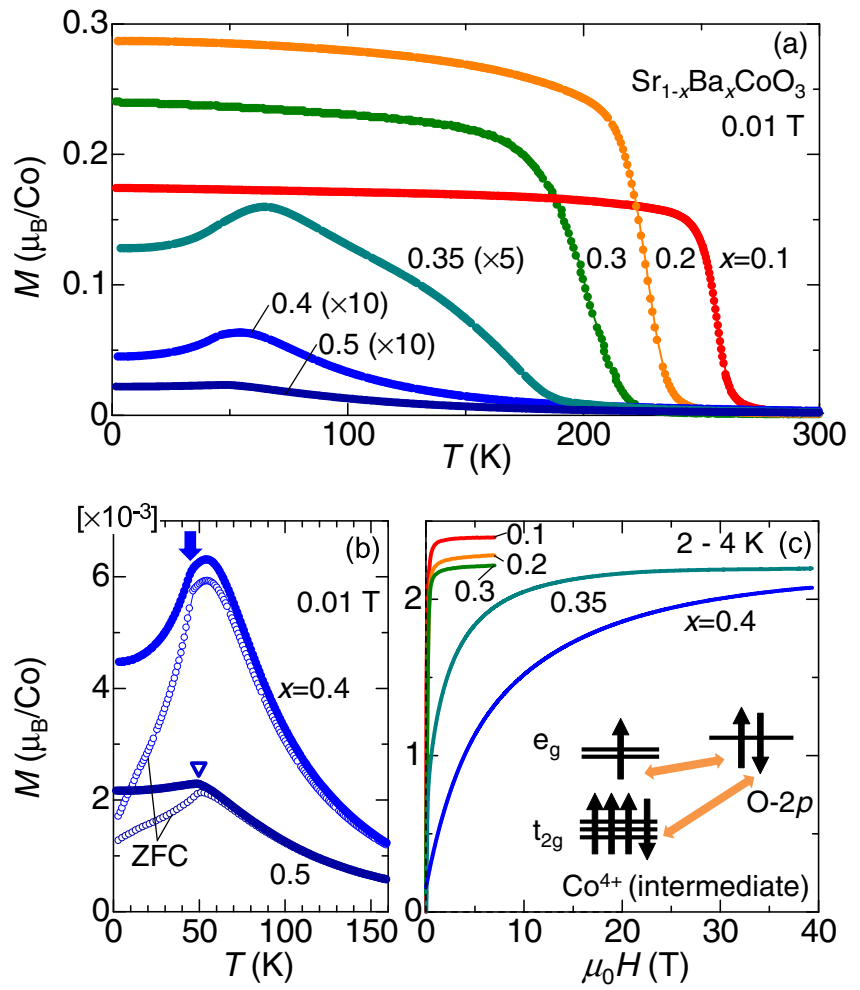

FIG. 2. (a) Temperature ( $T$ ) profiles of magnetization $(M)$ at $0.01 \mathrm{~T}$ for $\mathrm{Sr}_{1-x} \mathrm{Ba}_{x} \mathrm{CoO}_{3}(0.1 \leqslant x \leqslant 0.4)$ single crystals. (b) Low$T$ profiles of $M$ at $0.01 \mathrm{~T}$ for $x=0.4$ and 0.5 where the open circles denote the data in a warming run after zero-field cooling. (c) Field dependence of $M$ at the lowest temperature (2-4 K) for $x=0.1-0.4$. The profiles up to $\sim 40 \mathrm{~T}$ were measured with a pulsed magnet. The inset shows a schematic of the intermediate spin configuration of $\mathrm{Co}^{4+}$. The $d^{6} \underline{L}$ configuration ( $\underline{L}$ denotes an oxygen ligand hole) is likely to be stabilized by strong hybridization between the Co $3 d$ and the $\mathrm{O} 2 p$ orbitals.

0.1 to 0.35 , the ferromagnetic Curie temperature $T_{\mathrm{C}}$ [defined as the inflection point of $M(T)$ ] decreases systematically from 256 to $176 \mathrm{~K}$. Accordingly, the Weiss temperature $\theta$, determined by fitting the Curie-Weiss law to data above $250 \mathrm{~K}$ (Fig. S2 of the Supplemental Material [29]), also decreases in value comparable to $T_{\mathrm{C}}$. For $x=0.35$, the increase in $M$ below $T_{\mathrm{C}}$ is largely reduced, and instead a clear drop in $M$ upon cooling manifests itself at $T_{\mathrm{M}}[\sim 43 \mathrm{~K}$, defined as the peak position in $d M(T) / d T]$. For $x=0.4$, the ferromagnetic transition disappears, and the drop in $M$ is more conspicuous. As clearly seen in Fig. 2(b), the value of $M$ falls steeply below $T_{\mathrm{M}}$ in both measurements after the field- and zero-field-cooling runs. This behavior indicates that the compound with $x=0.4$ has an antiferromagneticlike ground state, although the observed positive value of $\theta \sim 170 \mathrm{~K}$ [Fig. 1(d)] implies that this state is unlikely to be a simple antiferromagnetic. Indeed, as shown later, it turns out to host helimagnetic correlations (see Fig. 4). For $x=0.5$, although an anomaly in $M$ is discernible around $50 \mathrm{~K}$, the steep drop in the field-cooling run seen for $x=0.4$ changes to a cusplike anomaly, suggesting a spin-glass-like transition. Figure 1(d) summarizes the overall magnetic phase diagram of $\mathrm{Sr}_{1-x} \mathrm{Ba}_{x} \mathrm{CoO}_{3}$ as functions of temperature and $x$, which reveals that the competition between ferromagnetism
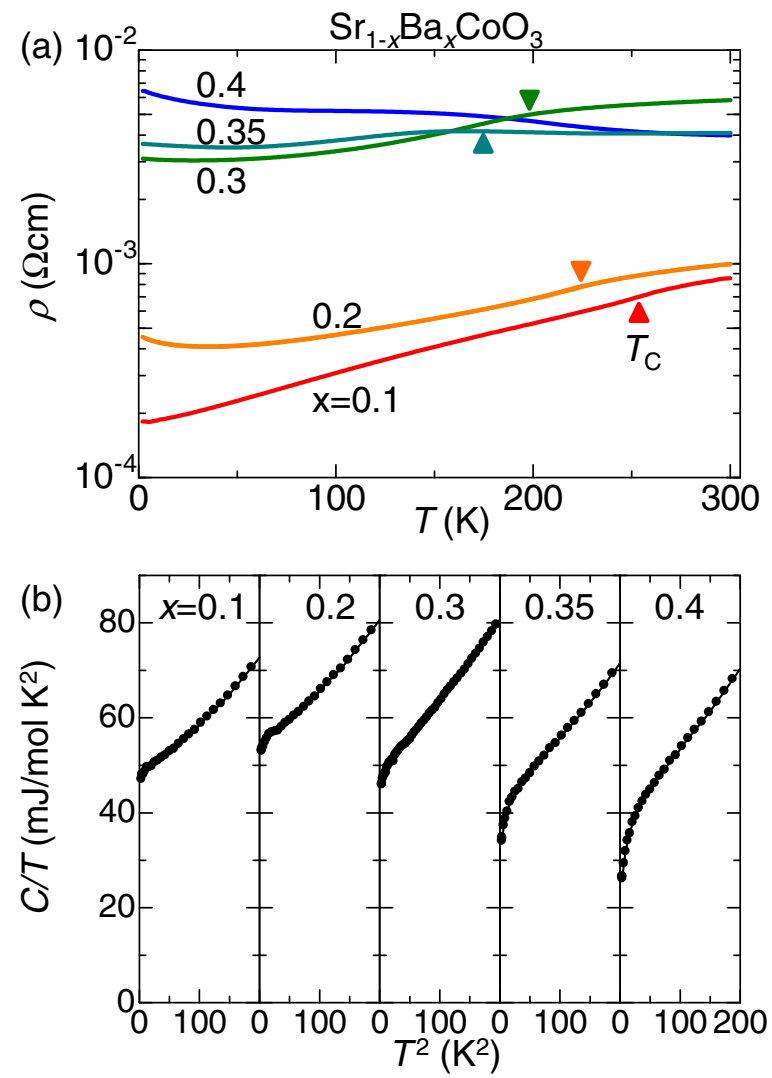

FIG. 3. (a) Temperature profile of resistivity $(\rho)$ at zero field for $\mathrm{Sr}_{1-x} \mathrm{Ba}_{x} \mathrm{CoO}_{3}(0.1 \leqslant x \leqslant 0.4)$ single crystals. The closed triangles denote the ferromagnetic transition temperature $T_{\mathrm{C}}$. (b) Specific heat divided by temperature $(C / T)$ versus $T^{2}$ for $0.1 \leqslant x \leqslant 0.4$.

and helimagnetism is sensitively dependent on the size of the cubic lattice, i.e., the Co-O bond length. In what follows, we will show the $x$ dependence of various physical properties for the present system.

To check the spin state of $\mathrm{Co}^{4+}$ ions upon Ba substitution, we have estimated the saturation magnetization $M_{\mathrm{S}}$ by measuring the field profile of $M$ at the lowest temperature (2-4 K) [Fig. 2(c)]. For $x=0.1-0.3$ with the ferromagnetic ground state, the $M$ value is saturated above $\sim 2 \mathrm{~T}$. However, the saturation field is substantially enhanced as $x$ increases to 0.35 where the helimagnetic instability sets in. Although the value of $M$ barely saturates at above $\sim 30 \mathrm{~T}$ for $x=0.35$, the magnetization does not reach $M_{\mathrm{S}}$ even under magnetic fields up to $40 \mathrm{~T}$ for $x \geqslant 0.4$. Figure 1(b) summarizes the value of $M_{\mathrm{S}}$ as a function of $x$. Despite a significant decrease in $T_{\mathrm{C}}$, the value of $M_{\mathrm{S}}$ is almost constant $\left(\sim 2.2-2.5 \mu_{B} / \mathrm{Co}\right)$ with $x$ up to 0.35 . The spin state thus appears to remain in a nearly intermediate, i.e., $S=3 / 2$ configuration [21], which is likely to be strongly hybridized with the ligand hole states [see the inset in Fig. 2(c)].

The temperature dependence of the resistivity for the $\mathrm{Sr}_{1-x} \mathrm{Ba}_{x} \mathrm{CoO}_{3}$ single crystals is shown in Fig. 3(a). The $x=$ 0.1 and 0.2 crystals show clear metallic behavior with resistivity lower than $1 \mathrm{~m} \Omega \mathrm{cm}$ similar to $\mathrm{SrCoO}_{3}$ [21], and a weak resistivity anomaly is discernible around the ferromagnetic transition as denoted by the closed triangles. With increasing 

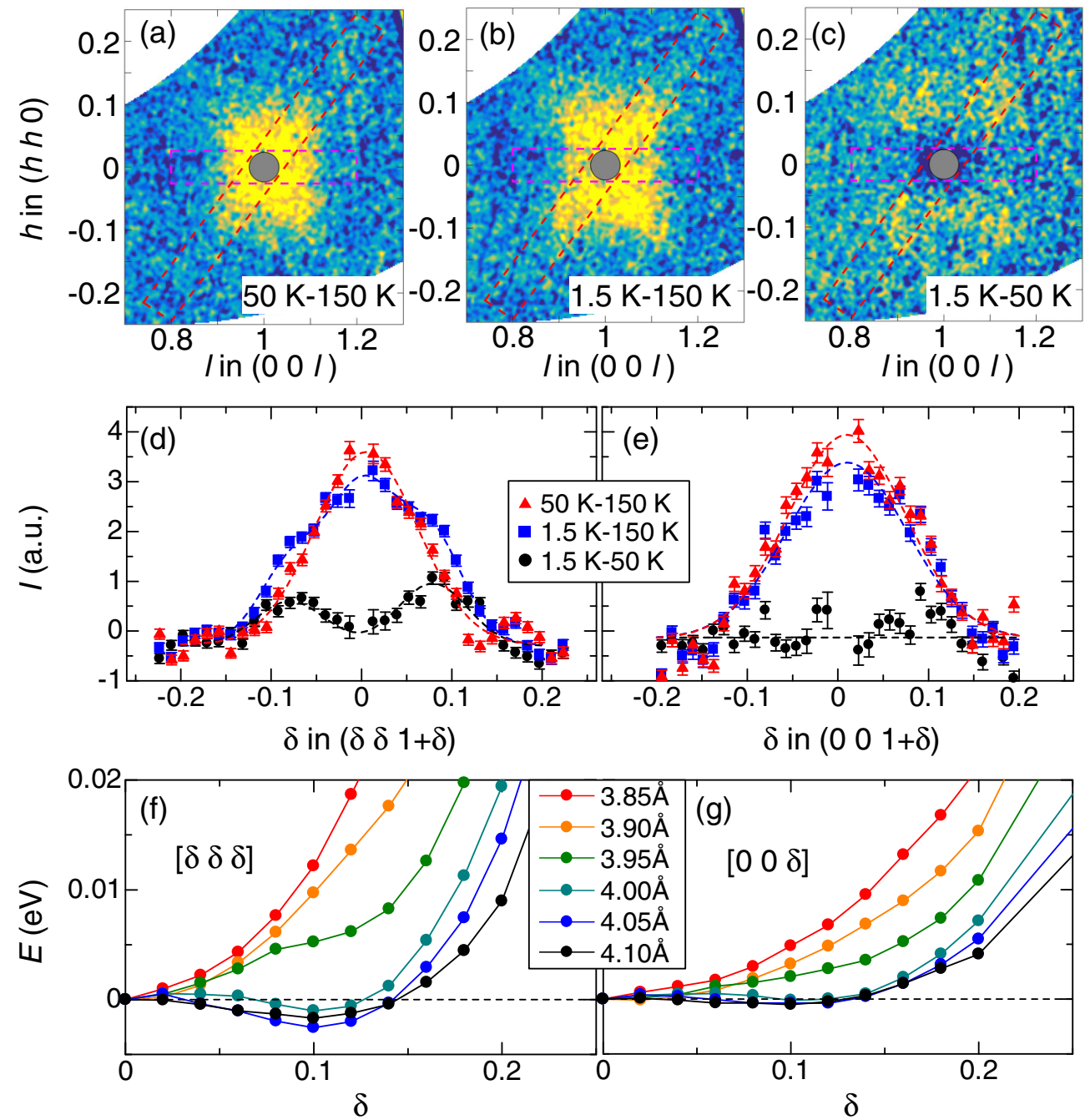

FIG. 4. Reciprocal-space maps of neutron-diffraction intensities for $x=0.4$ around the $\left(\begin{array}{lll}0 & 0 & 1\end{array}\right)$ reflection on the $(h h l)$ plane measured at (a) $50 \mathrm{~K}$ and at (b) and (c) $1.5 \mathrm{~K}$. To clearly show the magnetic scattering intensity, the intensities in panels (a)-(c) are plotted after the subtraction of those at 150,150 , and $50 \mathrm{~K}$, respectively. Line profiles of the intensity in panels (a)-(c) for cuts along the (d) $\langle 111\rangle$ and (e) $\left\langle\begin{array}{lll}0 & 0 & 1\end{array}\right\rangle$ directions. The cuts along the $\left.\left\langle\begin{array}{llll}1 & 1 & 1\end{array}\right\rangle\left(\begin{array}{lll}0 & 0 & 1\end{array}\right\rangle\right)$ directions were evaluated using the red (pink) boxes indicated in panels (a) $-(\mathrm{c})$. Calculated total energy as a function of magnetic propagation vector (f) $[\delta \delta \delta]$ and $(\mathrm{g})[00 \delta]$ for various lattice constants $(\AA)$ where the energy is plotted with respect to the energy of the FM state $E(\delta=0)$.

$x$, the resistivity increases at almost all temperatures and becomes less temperature dependent. The former indicates a crossover towards a high-resistivity state, which is consistent with a reduction in density of states at the Fermi energy for $x \geqslant 0.35$ signified by the specific-heat data [Fig. 3(b)]. On the other hand, the latter may result from scattering due to spin fluctuations persisting at low temperatures, which become enhanced by the competition between the ferromagnetic and the helimagnetic correlations. In addition, significant disorder effects due to both the $\mathrm{Sr} / \mathrm{Ba}$ solid solution and the oxygen vacancies may also play a role on such diffusive transport with high resistivity.

Results of specific-heat measurements shown in Figs. 3(b) and 1(c) suggest a significant change in density of states at the Fermi energy upon Ba substitution. For $x=0.1-0.3$ with a ferromagnetic ground state, the temperature profile of $C$ is reasonably well described by $C / T=\gamma+\beta T^{2}$, where $\gamma$ is the electronic specific-heat coefficient [Fig. 3(b)] [35]. The large $\gamma$ values of $45-50 \mathrm{~mJ} \mathrm{~mol}^{-1} \mathrm{~K}^{-2}$ for $x=0.1-0.3$ imply a moderately strong electron correlation, which is comparable to the $\gamma$ value of $\mathrm{SrCoO}_{3}$ reported previously [20]. For $x \geqslant$ 0.35 , the $C / T$ value plotted as a function of $T^{2}$ shows a clear downturn toward the lowest temperature, indicating a substantial decrease in the density of states at the Fermi energy. The $\gamma$ values estimated from $C / T$ are plotted as a function of $x$ in Fig. 1(c). Although the $\gamma$ value is more or less constant up to $x=0.3$, it steeply decreases for $x \geqslant 0.35$, indicating the density of states to become reduced in the helimagnetic state. The $\gamma$ value for $x=0.4\left(\sim 20 \mathrm{~mJ} \mathrm{~mol}^{-1} \mathrm{~K}^{-2}\right)$ is less than half those for $x=0-0.3$. Thus, a partial energy gap might be formed around the Fermi energy in the helimagnetic phase, being consistent with the large increase in resistivity for $x \geqslant 0.35$. Note here that, despite the large variation in the density of states upon $x$, the $M_{\mathrm{S}}$ value remains almost independent of $x$, which implies that the $M_{\mathrm{S}}$ value is mainly determined by the localized $t_{2 g}$ majority spins. In fact, 
according to a recent dynamical mean-field theory, it can be expected that the itinerant electronic states at the Fermi energy consist of both $e_{g}$ majority and $t_{2 g}$ minority spin states [36]. Since these two states have opposite spin states, they may provide a small contribution to $M_{\mathrm{S}}$.

Next we show results of neutron-diffraction measurements of the magnetic correlations for $x=0.4$. Figures 4(a)-4(c) show maps of the neutron-diffraction intensity around the (l $\left.\begin{array}{lll}0 & 0 & 1\end{array}\right)$ Bragg reflection for a single-crystalline sample at selected temperatures. The data in the panels (a) and (b) are plotted after subtraction of the data at $150 \mathrm{~K}$ in the paramagnetic (PM) state, which visualizes the magnetic scattering intensity around the $\left(\begin{array}{lll}0 & 0 & 1\end{array}\right)$ reflection. At $50 \mathrm{~K}$ just above $T_{\mathrm{M}}(\sim 44 \mathrm{~K})$, a nearly isotropic distribution of magnetic diffuse scattering is seen, which implies the presence of ferromagnetic correlations. At $1.5 \mathrm{~K}$ below $T_{\mathrm{M}}$, part of the magnetic scattering is observed to be anisotropically distributed, forming lobe shapes along the $\left\langle\begin{array}{llll}1 & 1 & 1\end{array}\right\rangle$ directions. The anisotropic distribution of the magnetic intensity that onsets below $T_{\mathrm{M}}$ can be identified by subtracting the neutrondiffraction intensity map at $50 \mathrm{~K}$ from that at $1.5 \mathrm{~K}$ as shown in Fig. 4(c). From this we find that incommensurate magnetic peaks are discernible at four equivalent positions along the $\langle 111\rangle$ directions. The presence of the incommensurate peaks is also clear from the line-cut profile of the intensity along 〈1 11 1) [Fig. 4(d)] with no intensity observed in the profile along the $\langle 001\rangle$ direction [Fig. 4(e)]. From a Gaussian fit to the line-cut profile, the peak position is determined to be $\boldsymbol{Q}=\left(\begin{array}{lll}0 & 0 & 1\end{array}\right) \pm(\delta \delta \delta)$ with $\delta=0.079(5)$ and most likely originates from incommensurately modulated helimagnetic correlations with a periodicity of approximately $28 \AA$. From the half-width at half maximum of the peaks the magnetic correlation length is estimated to be $12 \AA$. Such short-range correlation might reflect strong fluctuations induced by the presence of the competing ferromagnetic instability, which is consistent with the fact that $x=0.4$ is located near the ferromagnetic-helimagnetic phase boundary. In addition to this, disorder effects arising from the oxygen vacancies and solid solution may contribute to the short-range nature of the correlations. We have thus revealed that the ferromagnetic ground state in $\mathrm{Sr}_{1-x} \mathrm{Ba}_{x} \mathrm{CoO}_{3}$ tends towards incommensurate helimagnetism as $x$ increases from 0 to 0.4 .

The instability towards helimagnetic order in $\mathrm{Sr}_{1-x} \mathrm{Ba}_{x} \mathrm{CoO}_{3}$ can also be reproduced by first-principles calculations. Figures $4(\mathrm{f})$ and $4(\mathrm{~g})$ show the $\delta$ dependence of the total energy for various lattice constants and for the magnetic propagation vectors of $[\delta \delta \delta]$ and $[00 \delta]$, respectively. By increasing the lattice constant, the local minimum energy at $\delta \sim 0.1$ becomes the global minimum for [ $\delta \delta \delta$ ], indicating a first-order ferromagnetic-to-helimagnetic transition. Since the minimum energy for $[\delta \delta \delta]$ is lower than that for $[00 \delta]$, the propagation vector of the most stable helimagnetic order is determined to be $[\delta \delta \delta]$ with $\delta \sim 0.1$, which is close to the experimental result. The origin of the helimagnetic order may be attributed to a competition between the ferromagnetic double exchange interaction and the antiferromagnetic superexchange interaction [37,38], i.e., the former becomes weakened compared with the latter as the lattice expands. Such a tendency is, in fact, expected according to first-principles calculations performed for the perovskite-type ferrites that take into consideration a change in spin moment [38]. Alternatively, the hybridization of the oxygen $2 p$ orbital relevant to the negative $\Delta$ may play an important role by modulating the nature of the double exchange interaction $[13,17]$. It remains for a future theoretical work to reveal the detailed variation in exchange interactions and the mechanism of the helimagnetic transition upon lattice expansion.

\section{CONCLUSIONS}

In combination with first-principles calculations, this paper demonstrates that a simple cubic $\mathrm{Co}^{4+}-\mathrm{O}$ lattice has the potential to host incommensurate helical spin order that competes with the ferromagnetic order. The point here is that the stability of these phases in $\mathrm{Sr}_{1-x} \mathrm{Ba}_{x} \mathrm{CoO}_{3}$ can be controlled by the lattice size, i.e., the bandwidth. Therefore, it can be anticipated that the $\mathrm{Sr}_{1-x} \mathrm{Ba}_{x} \mathrm{CoO}_{3}$ thin-film heterostructures serve as a novel helimagnetic-based spintronics where the ferromagnetic and helimagnetic states are selectively stabilized by the epitaxial strain.

\section{ACKNOWLEDGMENTS}

The authors are grateful to M. Mostovoy for fruitful discussions. Neutron scattering was performed at the Swiss Neutron Source SINQ, Paul Scherrer Instutute (PSI). The work was, in part, supported by the JST PRESTO Hyper-nano-space design toward Innovative Functionality (Grant No. JPMJPR1412), the JSPS Grant-in-Aid for Scientific Research (A) Grant No. 17H01195, the Asahi Glass Foundation, the Swiss National Science Foundation through Grants No. 153451 and No. 166298, the Sinergia network on Nanoskyrmionics (Grant No. CRSII5-171003), and the European Research Council Project CONQUEST. E.C. acknowledges support from the Danish Research Council for Science and Nature through DANSCATT.
[1] S. W. Cheong and M. Mostovoy, Nat. Mater. 6, 13 (2007).

[2] Y. Tokura and S. Seki, Adv. Mater. 22, 1554 (2010).

[3] N. Nagaosa and Y. Tokura, Nat. Nanotechnol. 8, 899 (2013).

[4] T. Kimura, T. Goto, H. Shintani, K. Ishizaka, T. Arima, and Y. Tokura, Nature (London) 426, 55 (2003).

[5] M. Kenzelmann, A. B. Harris, S. Jonas, C. Broholm, J. Schefer, S. B. Kim, C. L. Zhang, S.-W. Cheong, O. P. Vajk, and J. W. Lynn, Phys. Rev. Lett. 95, 087206 (2005).
[6] T. Kimura, S. Ishihara, H. Shintani, T. Arima, K. T. Takahashi, K. Ishizaka, and Y. Tokura, Phys. Rev. B 68, 060403(R) (2003).

[7] H. Oda, Y. Yamaguchi, H. Takei, and H. Watanabe, J. Phys. Soc. Jpn. 42, 101 (1977).

[8] M. Reehuis, C. Ulrich, A. Maljuk, C. Niedermayer, B. Ouladdiaf, A. Hoser, T. Hofmann, and B. Keimer, Phys. Rev. B 85, 184109 (2012). 
[9] S. Ishiwata, M. Tokunaga, Y. Kaneko, D. Okuyama, Y. Tokunaga, S. Wakimoto, K. Kakurai, T. Arima, Y. Taguchi, and Y. Tokura, Phys. Rev. B 84, 054427 (2011).

[10] N. Hayashi, T. Yamamoto, H. Kageyama, M. Nishi, Y. Watanabe, T. Kawakami, Y. Matsushita, A. Fujimori, and M. Takano, Angew. Chem., Int. Ed. 50, 12547 (2011).

[11] N. Hayashi, T. Yamamoto, A. Kitada, A. Matsuo, K. Kindo, J. Hester, H. Kageyama, and M. Takano, J. Phys. Soc. Jpn. 82, 113702 (2013).

[12] T. Okubo, S. Chung, and H. Kawamura, Phys. Rev. Lett. 108, 017206 (2012).

[13] M. Azhar and M. Mostovoy, Phys. Rev. Lett. 118, 027203 (2017).

[14] S. Hayami, R. Ozawa, and Y. Motome, Phys. Rev. B 95, 224424 (2017).

[15] S. Ishiwata, T. Nakajima, J.-H. Kim, D. S. Inosov, N. Kanazawa, J. S. White, J. L. Gavilano, R. Georgii, K. Seemann, G. Brandl, P. Manuel, D. D. Khalyavin, S. Seki, M. Kinoshita, Y. Tokunaga, Y. Kaneko, Y. Taguchi, T. Arima, B. Keimer, and Y. Tokura, arXiv:1806.02309.

[16] A. E. Bocquet, A. Fujimori, T. Mizokawa, T. Saitoh, H. Namatame, S. Suga, N. Kimizuka, Y. Takeda, and M. Takano, Phys. Rev. B 45, 1561 (1992).

[17] M. Mostovoy, Phys. Rev. Lett. 94, 137205 (2005).

[18] P. Bezdicka, A. Wattiaux, J. C. Grenier, M. Pouchard, and P. Hagenmuller, Z. Anorg. Allg. Chem. 619, 7 (1993).

[19] M. Abbate, G. Zampieri, J. Okamoto, A. Fujimori, S. Kawasaki, and M. Takano, Phys. Rev. B 65, 165120 (2002).

[20] S. Balamurugan, K. Yamaura, A. B. Karki, D. P. Young, M. Arai, and E. Takayama-Muromachi, Phys. Rev. B 74, 172406 (2006).

[21] Y. Long, Y. Kaneko, S. Ishiwata, Y. Taguchi, and Y. Tokura, J. Phys.: Condens. Matter 23, 245601 (2011).

[22] H. Xia, J. Dai, Y. Xu, Y. Yin, X. Wang, Z. Liu, M. Liu, M. A. McGuire, X. Li, Z. Li, C. Jin, Y. Yang, J. Zhou, and Y. Long, Phys. Rev. Mater. 1, 024406 (2017).
[23] T. Osaka, H. Takahashi, H. Sagayama, Y. Yamasaki, and S. Ishiwata, Phys. Rev. B 95, 224440 (2017).

[24] R. H. Potze, G. A. Sawatzky, and M. Abbate, Phys. Rev. B 51, 11501 (1995).

[25] T. Saitoh, T. Mizokawa, A. Fujimori, M. Abbate, Y. Takeda, and M. Takano, Phys. Rev. B 55, 4257 (1997).

[26] K. Asai, A. Yoneda, O. Yokokura, J. M. Tranquada, G. Shirane, and K. Kohn, J. Phys. Soc. Jpn. 67, 290 (1998).

[27] Y. W. Long, Y. Kaneko, S. Ishiwata, Y. Tokunaga, T. Matsuda, H. Wadati, Y. Tanaka, S. Shin, Y. Tokura, and Y. Taguchi, Phys. Rev. B 86, 064436 (2012).

[28] H. Sakai, S. Ishiwata, D. Okuyama, A. Nakao, H. Nakao, Y. Murakami, Y. Taguchi, and Y. Tokura, Phys. Rev. B 82, 180409(R) (2010).

[29] See Supplemental Material at http://link.aps.org/supplemental/ 10.1103/PhysRevMaterials.2.104412 for the characterization of oxidized single crystals and Curie-Weiss plots.

[30] J. P. Perdew, K. Burke, and M. Ernzerhof, Phys. Rev. Lett. 77, 3865 (1996).

[31] G. Kresse and D. Joubert, Phys. Rev. B 59, 1758 (1999).

[32] D. Hobbs, G. Kresse, and J. Hafner, Phys. Rev. B 62, 11556 (2000).

[33] L. M. Sandratskii, Phys. Status Solidi B 136, 167 (1986).

[34] H. Sakai, J. Fujioka, T. Fukuda, D. Okuyama, D. Hashizume, F. Kagawa, H. Nakao, Y. Murakami, T. Arima, A. Q. R. Baron, Y. Taguchi, and Y. Tokura, Phys. Rev. Lett. 107, 137601 (2011).

[35] A slight deviation from the straight line might arise from the magnon contribution in the ferromagnetically ordered phase. The $\gamma$ value was estimated by extrapolating the $C / T$ data close to the lowest temperature.

[36] J. Kuneš, V. Křápek, N. Parragh, G. Sangiovanni, A. Toschi, and A. V. Kozhevnikov, Phys. Rev. Lett. 109, 117206 (2012).

[37] Z. Li, R. Laskowski, T. Iitaka, and T. Tohyama, Phys. Rev. B 85, 134419 (2012)

[38] Z. Li, T. Iitaka, and T. Tohyama, Phys. Rev. B 86, 094422 (2012). 